ARTIGO ORIGINAL ORIGINAL ARTICLE

Palavras-chave:

economia da saúde, política de saúde, modelo Logit

\section{Análise dos determinantes da utilização do Programa Farmácia Popular do Brasil}

\author{
Analysis of the determinants in the use of the \\ Popular Pharmacy Program in Brazil \\ Pietro André Telatin Paschoalino', José Luiz Parré2, \\ Cássia Kely Favoretto Costa², Wander Plassa ${ }^{3}$, José Rodrigo Gobi' \\ DOI: $10.21115 / J B E S . v 13 . n 1 . p 2-13$
}

\section{RESUMO}

Objetivo: $O$ estudo analisa os determinantes de o indivíduo participar do Programa Farmácia Popular do Brasil (PFPB). Métodos: Utilizou-se a base de microdados da Pesquisa Nacional de Saúde em 2013, empregando o modelo Logit. Resultados: Observou-se que, conforme a idade do indivíduo aumenta, maior a probabilidade do uso de medicamentos pelo programa. Ademais, ter maior escolaridade, pertencer à classe de renda alta e possuir planos de saúde diminui essa probabilidade. Verificou-se, ainda, que a percepção de saúde de cada indivíduo não altera a probabilidade de uso do PFPB, mas o diagnóstico de doenças crônicas é importante fator explicativo para o uso de medicamentos. Conclusão: A utilização do programa está relacionada com fatores socioeconômicos e de morbidade, demonstrando a importância de políticas de assistência farmacêutica no processo saúde/doença, principalmente para indivíduos inseridos em baixas classes de renda e com doença crônica ou de longa duração. Os resultados permitem que os gestores públicos tenham uma maior compreensão do uso do programa pela população, possibilitando melhor direcionamento, ampliação e qualificação do acesso aos medicamentos.

\begin{abstract}
Objective: The study analyzes the determinants of the individual who participate in the Popular Pharmacy Program in Brazil (PFPB). Methods: It used a microdata base from the National Health Survey in 2013, using the Logit model. Results: Observe if the individual's age increases, the more likely the program is to use medication. In addition, greater schooling, belonging to the highincome class and using health plans decrease this probability. It was also found that the perception of health of each individual does not change the probability of using PFPB, but the diagnosis of chronic diseases is an important explanatory factor for the use of medicines. Conclusion: The use of the program is related to socioeconomic and morbidity factors, demonstrating the importance of pharmaceutical assistance policies in the health/disease process, especially for individuals in lowincome classes and with chronic or long-term illness. The results allow public managers to have a greater understanding the use of the program by the population, enabling better targeting, expansion and qualification of access to medicines.
\end{abstract}

\footnotetext{
Recebido em: 20/11/2020. Aprovado para publicação em: 22/04/2021.

1. Doutorando do Programa de Pós-Graduação em Ciências Econômicas da Universidade Estadual de Maringá (UEM), Maringá, PR, Brasil.

2. Docente Doutor do Programa de Pós-Graduação em Ciências Econômicas e do Departamento de Economia da Universidade Estadual de Maringá (UEM), Maringá, PR, Brasil.

3. Doutorando do Programa de Pós-Graduação em Economia Aplicada da Universidade de São Paulo (FEA-RP), Ribeirão Preto, SP, Brasil.

Instituição onde o trabalho foi executado: Universidade Estadual de Maringá, Maringá, PR, Brasil.

Financiamento: Não houve.

Conflito de interesse: Não houve.

Autor correspondente: Pietro André Telatin Paschoalino. E-mail: pietropasch@gmail.com
} 


\section{Introdução}

O Programa Farmácia Popular do Brasil (PFPB) foi criado com o objetivo de oferecer mais uma alternativa de acesso aos medicamentos considerados essenciais. Atualmente, com o nome "Aqui tem Farmácia Popular", o programa funciona por meio do credenciamento de farmácias e drogarias comerciais, aproveitando a dinâmica da cadeia farmacêutica (produção x distribuição x varejo). São oferecidos medicamentos gratuitos para hipertensão, diabetes e asma, além de medicamentos com até $90 \%$ de desconto (Brasil, 2020b).

O PFPB já atendeu mais de 47 milhões de brasileiros até novembro de 2017. Os atendimentos mensais no programa foram de cerca de 10 milhões de pessoas em 2017, beneficiando, principalmente, as pessoas com 60 anos ou mais. Além disso, no último ano destacado, o número de estabelecimentos chegou a mais de 30 mil, distribuídos em 4.338 municípios brasileiros (Brasil, 2018b).

Apesar de o perfil socioeconômico da população ter sido o critério inicial para a definição da abrangência do programa, ou seja, da parcela da população que tem dificuldades para arcar com custos de tratamento devido ao impacto no orçamento familiar, a gratuidade dos medicamentos não faz distinção entre indivíduos de baixa ou alta renda. Assim, apesar de o foco ser indivíduos que poderiam abandonar o tratamento de suas doenças devido a restrições orçamentárias, qualquer pessoa pode participar do programa por meio da simples apresentação de receituário médico (Brasil, 2005).

Segundo Conass (2016), o PFPB apresentou alguns resultados positivos sobre alguns indicadores na área da saúde, como redução de $20 \%$ das internações hospitalares por hipertensão e de 16\% por crise asmática após a implantação da gratuidade desses medicamentos. Além disso, os benefícios sociais gerados pelo programa devem ser maiores quando utilizado por participantes de baixa renda, pois eles demandam mais serviços (internações, tratamentos, entre outros) no Sistema Único de Saúde (SUS).

Diante do exposto, o objetivo deste artigo é analisar os determinantes da probabilidade de o indivíduo participar do PFPB. Para tal, utilizou-se o modelo Logit em duas diferentes especificações: uma que considera, além de características individuais, de renda, educacionais e domiciliares, uma característica importante relacionada a estado de saúde, qual seja, ter ou não sido diagnosticado com doença crônica; e outra que considera, além das variáveis supracitadas, as respostas subjetivas de status de saúde (saúde boa, regular ou ruim). Para essas estimações, são utilizadas informações coletadas da Pesquisa Nacional de Saúde (PNS) de 2013.

\section{Revisão de literatura}

\section{Revisão teórica}

O PFPB foi criado em 13 abril de 2004, pela Lei no 10.858, e regulamentado pelo Decreto no 5.090, de 20 de maio de 2004 (Brasil, 2004). As primeiras unidades próprias foram inauguradas em junho de 2004 nas cidades de Salvador, Rio de Janeiro, São Paulo e Goiânia. A meta era assegurar medicamentos essenciais para o tratamento dos agravos com maior incidência na população, mediante redução do seu custo (Pereira, 2013).

Inicialmente, esse programa previa exclusivamente a instalação de farmácias denominadas rede própria (modelo 1), em parceria com estados e municípios, para a dispensação desses medicamentos. As unidades próprias são operacionalizadas de forma centralizada pela Fundação Oswaldo Cruz (Fiocruz), que coordena sua estruturação e executa a compra dos medicamentos, o abastecimento, a distribuição e a capacitação dos profissionais (Pereira, 2013).

O programa foi caracterizado pela gestão conjunta entre Ministério da Saúde e Fiocruz, que passou a cumprir o papel de executora do programa. Foi lançado como a primeira iniciativa federal de copagamento para acesso a medicamentos, mediante ressarcimento à Fiocruz. O programa foi pensado como complemento à dispensação dos medicamentos cobertos pelas Farmácias Básicas, que são unidades dispensadoras do SUS na atenção básica (Almeida et al., 2019). As unidades próprias contavam com um elenco de 112 itens, entre medicamentos e o preservativo masculino, com redução de até 90\% do valor de mercado (Brasil, 2020b).

Em dezembro de 2004, foi publicada a Portaria no 2.587 e, com base no Decreto no 5.090/2004, as unidades próprias (farmácias populares) foram ampliadas por meio de parcerias com os estados e municípios, instituições da área de saúde e de ensino superior sem fins lucrativos. Essa etapa foi denominada de modelo 2 e manteve os mesmos critérios da primeira, mas, na prática, não houve diferenças para a população. A diferença entre os modelos é na gestão, já que o modelo 2 se baseia na gestão conjunta da Fiocruz com parceiros, enquanto o modelo 1 era sem parceiros (Santos-Pinto et al., 2011).

Em março de 2006, por meio da Portaria GM no 491, o Farmácia Popular foi expandido com o lançamento de um sistema de copagamento desenvolvido em parceria com farmácias e drogarias privadas. Foi desenvolvida, assim, uma nova versão desse programa, o "Aqui tem Farmácia Popular", que propôs garantir a ampliação do acesso a medicamentos essenciais, indicados em tratamentos de doenças com grande prevalência na população (Brasil, 2006). No sistema de copagamento, o governo paga um valor fixo pelos medicamentos e o cidadão paga a diferença, variando esse valor de acordo com cada produto (Conass, 2016).

A partir de 2011, com a campanha "Saúde não tem preço", houve a retirada de copagamento em alguns casos, ou seja, o governo arca com o valor de referência dos medicamentos, que paga diretamente aos estabelecimentos credenciados. A medida incluía inicialmente apenas anti-hipertensivos e antidiabéticos, e posteriormente contemplou antiasmáticos (Silva \& Caetano, 2015).

Em relação ao valor transferido pelo Ministério da Saúde, em 2006 os custos referentes à ação somaram mais de 
$\mathrm{R} \$ 34.723 .571,00$, chegando em 2010 a R\$ 247.220.802,00. Com o início das ações de gratuidade pela campanha "Saúde não tem preço", a transferência de valor aos estabelecimentos cadastrados atingiu a cifra de R\$ 831.085.837,00, e para o ano de 2017 o recurso investido foi de $\mathrm{R} \$ 2.815 .000 .000,00$ (Brasil, 2018b).

O programa atingiu, em 2018, cerca de 4.338 municípios brasileiros, sendo essa grande rede de distribuição um dos possíveis fatores de sucesso para a melhora dos indicadores citados (reduções de internações hospitalares por hipertensão e crise asmática). Atualmente, o programa funciona tanto no sistema de copagamentos como pela oferta de medicamentos gratuitos (Brasil, 2020b).
A utilização das duas vertentes para provisionamento dos medicamentos à população acaba em 2017, com o fim da rede própria (Almeida et al., 2019). Após a decisão pelo fim do repasse de manutenção às unidades de rede própria do PFPB, ficou decidido que o Ministério da Saúde iria ampliar os recursos destinados para estados e municípios na compra dos medicamentos do Componente Básico da Assistência Farmacêutica, e o valor enviado mensalmente para a compra passaria de $\mathrm{R} \$ 5,10$ por habitante para $\mathrm{R} \$ 5,58$, atualizando a população da base de cálculo para IBGE 2016 (Conasems, 2017). A Tabela 1 realiza uma síntese dos medicamentos disponibilizados pelo "ATFP", atualizado em 06/03/2018.

Tabela 1. Lista de medicamentos disponibilizados pelo "Aqui Tem Farmácia Popular"

\begin{tabular}{|c|c|c|}
\hline Medicamento & Indicação & Gratuidade \\
\hline Atenolol 25 mg & Hipertensão & Gratuidade \\
\hline Captopril 25 mg & Hipertensão & Gratuidade \\
\hline Cloridrato de propranolol $40 \mathrm{mg}$ & Hipertensão & Gratuidade \\
\hline Hidroclorotiazida 25 mg & Hipertensão & Gratuidade \\
\hline Losartana potássica 50 mg & Hipertensão & Gratuidade \\
\hline Maleato de enalapril $10 \mathrm{mg}$ & Hipertensão & Gratuidade \\
\hline Glibenclamida 5 mg & Diabetes & Gratuidade \\
\hline Cloridrato de metformina 500 mg & Diabetes & Gratuidade \\
\hline Cloridrato de metformina 500 mg - ação prolongada & Diabetes & Gratuidade \\
\hline Cloridrato de metformina 850 mg & Diabetes & Gratuidade \\
\hline Insulina humana $100 \mathrm{UI} / \mathrm{mL}$ & Diabetes & Gratuidade \\
\hline Insulina humana regular $100 \mathrm{UI} / \mathrm{mL}$ & Diabetes & Gratuidade \\
\hline Sulfato de salbutamol 5 mg & Asma & Gratuidade \\
\hline Sulfato de salbutamol $100 \mathrm{mcg}$ & Asma & Gratuidade \\
\hline Dipropionato de beclometasona 50 mcg & Asma & Gratuidade \\
\hline Dipropionato de beclometasona 200 mcg/dose & Asma & Gratuidade \\
\hline Dipropionato de beclometasona 200 mcg/cápsula & Asma & Gratuidade \\
\hline Dipropionato de beclometasona $250 \mathrm{mcg}$ & Asma & Gratuidade \\
\hline Brometo de ipratrópio 0,25 mg/mL & Asma & Gratuidade \\
\hline Brometo de ipratrópio 0,02 mg/dose & Asma & Gratuidade \\
\hline Acetato de medroxiprogesterona $150 \mathrm{mg}$ & Anticoncepção & Copagamento \\
\hline Etinilestradiol 0,03 mg + levonorgestrel 0,15 mg & Anticoncepção & Copagamento \\
\hline Noretisterona 0,35 mg & Anticoncepção & Copagamento \\
\hline Valerato de estradiol $5 \mathrm{mg}+$ enantato de noretisterona $50 \mathrm{mg}$ & Anticoncepção & Copagamento \\
\hline Sinvastatina $10 \mathrm{mg}$ & Dislipidemia & Copagamento \\
\hline Sinvastatina $20 \mathrm{mg}$ & Dislipidemia & Copagamento \\
\hline Sinvastatina $40 \mathrm{mg}$ & Dislipidemia & Copagamento \\
\hline Budesonida $32 \mathrm{mcg}$ & Rinite & Copagamento \\
\hline Budesonida $50 \mathrm{mcg}$ & Rinite & Copagamento \\
\hline Dipropionato de beclometasona 50 mcg & Rinite & Copagamento \\
\hline Carbidopa 25 mg + levodopa 250 mg & Doença de Parkinson & Copagamento \\
\hline Cloridrato de benserazida 25 mg + levodopa 100 mg & Doença de Parkinson & Copagamento \\
\hline Alendronato de sódio 70 mg & Osteoporose & Copagamento \\
\hline Maleato de timolol 2,5 mg & Glaucoma & Copagamento \\
\hline Maleato de timolol $5 \mathrm{mg}$ & Glaucoma & Copagamento \\
\hline
\end{tabular}


Considerando as características e o funcionamento do PFPB, uma maneira teórica de compreendê-lo é internalizar a externalidade positiva gerada no oferecimento de medicamentos (ótica do subsídio governamental). Com o aumento da distribuição dos medicamentos, haveria diminuição de internações e óbitos, que gera melhora de bem-estar tanto para os indivíduos, que apresentariam melhor estado de saúde, quanto para o governo (economia de recursos com a diminuição das internações). Assim, são justificadas a implementação e a utilização do programa por parte do governo. A utilização do subsídio poderia ser resumida por meio da Figura 1.

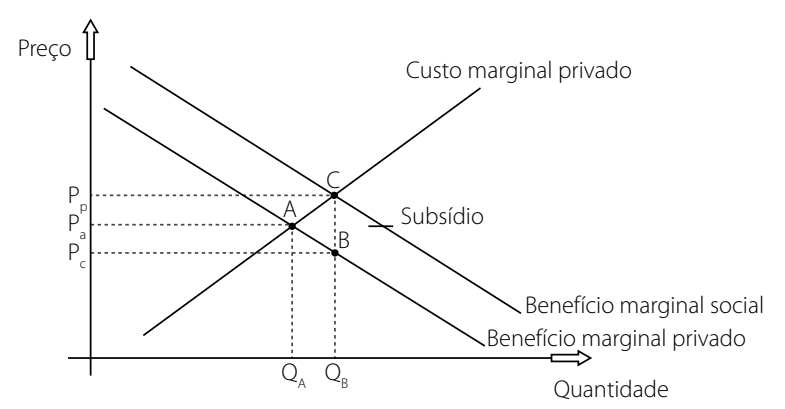

Fonte: Adaptada de Arvate \& Biderman (2013).

Figura 1. Efeito da implementação de um subsídio quando existe externalidade positiva

$\mathrm{Na}$ internalização de uma externalidade (Figura 1), o Benefício Marginal Social é maior que o Benefício Marginal Privado (curva de demanda). Sem o subsídio, a quantidade e o preço de equilíbrio correspondem a $Q_{A}$ e $P_{A^{\prime}}$ respectivamente. Com o subsídio, a produção total aumenta para $\mathrm{Q}_{\mathrm{B}^{\prime}}$ e o preço recebido pelo produtor é $P_{p_{i}}$ já o preço pago pelo consumidor é $P_{C}$ (menor que $P_{A}$ ). $O$ subsídio pago pelo governo corresponde à área $Q_{B} P_{P} P_{c^{\prime}}$ refletindo a quantidade consumida multiplicada pelo subsídio por unidade transacionada $\left(\mathrm{P}_{\mathrm{p}}-\mathrm{P}_{\mathrm{c}}\right)$.

Dessa forma, como a utilização dos medicamentos gera bem-estar para toda a sociedade, é justificada a utilização do subsídio governamental oferecido pelo governo para a população. Assim, atividades que proporcionam externalidades positivas devem ser incentivadas (Arvate \& Biderman, 2013).

Porém, cabe destacar que, apesar de a gratuidade se referir a medicamentos essenciais, teoricamente, os benefícios sociais em oferecê-los para a população de camadas sociais mais baixas seriam maiores, pois esses indivíduos tenderiam a utilizar o SUS a partir de alguma complicação de saúde, o que acaba por acarretar maiores gastos para o governo. Por sua vez, indivíduos de classe social mais alta poderiam utilizar plano de saúde ou arcar com os custos privados, por exemplo, de internações e consultas médicas.

Assim, por hipótese, quanto maior a proporção de indivíduos de baixa renda entre os utilizadores do programa, maior o benefício social alcançado pelo governo. Destaca-se que, para a utilização do programa, não é necessária nenhuma comprovação de renda, apenas apresentar um documento oficial com foto e o número do Cadastro Pessoa Física (CPF), ou documento de identidade em que conste o número do CPF, assim como uma receita médica dentro do prazo de validade (tanto do SUS quanto particular) (Brasil, 2020a).

\section{Evidências empíricas}

Na literatura científica existem diversos estudos que analisam o PFPB. Empiricamente, há relativa carência de trabalhos com enfoque econômico sobre o tema. Verifica-se que a maioria dos estudos avalia os resultados apenas de forma descritiva, o que, por um lado, faz com que o presente estudo possa contribuir significativamente com a literatura. Dos trabalhos que tentam avaliar os programas, mesmo que alguns apenas de maneira descritiva, destacam-se: de Santos-Pinto et al. (2011), Motta et al. (2013), Costa et al. (2016), Garcia et al. (2017) e Almeida et al. (2019).

Santos-Pinto et al. (2011) analisam o modelo inicial do PFPB, baseado na implantação das unidades de dispensação de medicamentos, por meio de dados obtidos por entrevistas com atores-chave. Nessa fase inicial do programa, ainda não eram oferecidos medicamentos gratuitos, e o público-alvo do programa eram indivíduos de classe média baixa que teriam alguma possibilidade de arcar com os medicamentos. De fato, verificou-se que os medicamentos oferecidos pelo programa estavam tomando o lugar dos gratuitos, oferecidos pelo SUS, principalmente na região Norte e Nordeste. Uma possível explicação para tal fenômeno pode estar relacionada à presença contínua dos medicamentos e bom atendimento, diferentemente do apresentado pelo SUS. Esse crescimento do programa relativamente aos oferecidos diretamente pelo SUS pode ter sido um dos motivos para a sua ampliação com a inclusão dos medicamentos gratuitos.

Por sua vez, Motta et al. (2013) analisam o PFPB sobre aspecto de componente da despesa. A partir da metodologia de Equilíbrio Geral Computável, calibrado para o ano de 2005 de acordo com a matriz insumo-produto brasileira, avaliam seu impacto sobre o crescimento econômico e do consumo das famílias (ambos positivos), além de verificarem que o aumento de bem-estar das famílias ocorre nos decis inferiores de renda.

Costa et al. (2016) descrevem a proporção de hipertensos e diabéticos maiores de 18 anos que referiram obter medicamentos para o controle dessas doenças no PFPB. Para isso, realizaram estudo descritivo da base de dados da PNS 2013. Os dados mostraram que cerca de um terço dos indivíduos hipertensos e mais da metade dos diabéticos obtiveram pelo menos um medicamento no programa. Destaque para indivíduos de cor preta e com menor nível de escolaridade. Verificaram, ainda, que, para a amostra analisada, o PFPB atingiu parcelas importantes da população brasileira, especialmente no segmento socioeconômico mais vulnerável.

Garcia et al. (2017) comparam em uma análise de eficiência por meio de simulações de Monte Carlo, o programa 
Rede Farmácia de Minas Gerais (RFMG) e o PFPB, com dados coletados de várias fontes entre janeiro e agosto de 2013. A avaliação econômica realizada demonstra que investimentos em um modelo público integrado com os princípios e as diretrizes do SUS são mais adequados para garantir assistência farmacêutica integral e universal com qualidade para a população. Assim, a aplicação dos serviços de assistência farmacêutica diretamente na rede pública é economicamente mais eficiente do que a terceirização dos serviços para o setor privado no PFPB. No entanto, ressalta-se que essas unidades de assistência apresentaram baixa produtividade, o que afeta diretamente o custo médio dos serviços prestados.

Por fim, Almeida et al. (2019) objetivam avaliar o impacto do PFPB sobre a saúde de pacientes crônicos (diabetes e hipertensão), medido pelas taxas de internação e mortalidade. Para isso, utilizaram o modelo estatístico de diferenças em diferenças com efeito fixo. Os autores utilizam dados de 5.566 municípios, referentes ao período de 2003 a 2016, com dados coletados de várias fontes. De acordo com os resultados dos autores, tanto o quantitativo das internações hospitalares quanto o de óbitos devidos a hipertensão e diabetes foram reduzidos pela ampliação ao acesso aos seus medicamentos, e a rede conveniada foi que induziu a tais resultados. Por fim, verificaram ainda evidências de transbordamento espacial e manutenção dos impactos sobre diferentes grupos etários, com destaque para os idosos.

\section{Métodos}

\section{Método de estimação}

Para estimar os determinantes da utilização dos benefícios do PFPB, utilizou-se o modelo Logit. O intuito da utilização dessa abordagem é avaliar as probabilidades de ocorrência de determinado evento (Greene, 2011). Para o proposto estudo, buscou-se avaliar as probabilidades de o indivíduo utilizar $\left(Y_{i}=1\right)$ ou não utilizar o programa $\left(Y_{i}=0\right)$.

De acordo com Cameron \& Trivedi (2005), o modelo de regressão é formado pela parametrização da probabilidade $p$ de depender de um $\beta$ vetor de regressores $x$ e de um vetor com parâmetros $K \times 1$. A partir do modelo com probabilidade acumulada dada por:

$$
\operatorname{Prob}\left(Y_{i}=1 \mid \mathbf{x}\right)=\tilde{F}\left(x_{i}^{\prime} \beta\right)
$$

em que $x$ é o vetor de variáveis explicativas e $\beta$ é o conjunto de parâmetros relacionados às variáveis explicativas que reflete o impacto da mudança em $x$ na probabilidade de $Y$; o modelo Logit surge quando $F(\cdot)$ é a função de distribuição acumulada logística. A equação (1) também pode ser interpretada por meio de:

$$
\operatorname{Prob}(Y=1 \mid x)=\frac{e^{x^{\prime} \beta}\left(x^{\prime} \beta\right)}{1+e^{x^{\prime} \beta}}=\Lambda\left(x^{\prime} \beta\right)
$$

em que $\Lambda(\cdot)$ representa a função de distribuição acumulada logística. Para verificar os efeitos marginais no modelo Logit, é necessário diferenciar a equação (2), ou seja:

$$
\frac{\partial \wedge\left(x^{\prime} \beta\right)}{\partial x}=\Lambda\left(x^{\prime} \beta\right)\left[1-\Lambda\left(x^{\prime} \beta\right)\right] \beta
$$

Segundo Plassa e Cunha (2016), os efeitos marginais são utilizados para verificar a variação percentual da probabilidade de o evento ocorrer quando uma variável independente é alterada. Destaca-se que sinal do coeficiente apresentará o sinal do efeito marginal, desde que $F(\cdot)>0$ Cameron \& Trivedi, 2005).

\section{Fonte e descrição dos dados}

A base de dados utilizada no presente estudo para avaliação dos determinantes da utilização do PFPB foi a PNS de 2013, que dispõe dos microdados mais atualizados sobre o tema. Além disso, cabe destacar que, após modificação da base de dados, a amostra final foi composta de 9.257 indivíduos. Dentre as mudanças realizadas para a obtenção da amostra afinal, excluíram-se os indivíduos que não responderam às perguntas: se obtiveram medicamento por meio do PFPB, e indivíduos com idade menor que 18 anos. Também foram eliminados da amostra os indivíduos que não apresentaram renda per capita positiva para nenhum dos moradores do domicílio, estavam inseridos em raças pouco representativas (indígenas e amarelos, ficando na amostra apenas as raças branca, preta e parda), apresentaram renda per capita ${ }^{1}$ superior a 50 mil (devido à baixa representatividade) ou não eram chefes do domicílio ou cônjuges de chefes, de sexo oposto.

Assim, destaca-se que a amostra é selecionada para um grupo específico da população, maiores de 18 anos e chefes/ cônjuges e que responderam às variáveis de interesse. Com relação às variáveis utilizadas no modelo Logit, realizaram-se duas especificações. Na primeira, foram consideradas características sociodemográficas, renda e avaliação de saúde e de região. Assim, o modelo empírico pode ser descrito por:

$$
Y_{i}=\beta_{1} X_{i}^{1}+\beta_{2} X_{i}^{2}+\beta_{3} X_{i}^{3}+\beta_{4} X_{i}^{4}+\mu_{t}
$$

em que a variável dependente $Y_{i}$ é igual a 1 caso o indivíduo tenha utilizado o PFPB, e igual a 0, caso contrário. Essa variável foi construída para indivíduos que tiveram receitado algum medicamento após a utilização de serviços de saúde e se algum dos medicamentos foi obtido no PFPB.

A variável $X_{i}^{1}$ é um vetor de características sociodemográficas dos indivíduos (idade, raça², gênero, número de moradores, além do nível de instrução $)$. Já $X_{i}^{2}$ é um vetor

1 Renda per capita construída pela soma da renda do trabalho, rendimento bruto mensal de outros trabalhos, valor em $\mathrm{R} \$$ de aposentadorias ou pensões, pensão alimentícia ou doação em dinheiro de pessoas que não moravam no domicílio, juros de aplicações financeiras, dividendos, programas sociais, seguro-desemprego e outros rendimentos, além de valor recebido em $\mathrm{R} \$$ em aluguéis e arrendamentos, dividido pelo número de moradores de cada domicílio.

2 Negros foram omitidos na análise, uma vez que foram escolhidos como referência de comparação.

3 Classe sem instrução foi omitida. 
de variáveis de renda (classe de renda baixa, média, alta ${ }^{4}$ e utilização de plano de saúde). Para definição das classes de renda, utilizou-se a distribuição amostral final.

A variável $X_{i}^{3}$ refere-se ao vetor de variável relacionada à avaliação de saúde (diagnosticado com doença crônica). Por fim, $X_{i}^{4}$ é o vetor de regiões (Sul, Sudeste, Centro-Oeste e Norte ${ }^{5}$ ) e domiciliares, como número de moradores e se a residência se encontra na região urbana ou rural.

A segunda especificação inclui no vetor $X_{i}^{3}$ variáveis de avaliação de saúde, sendo essas variáveis subjetivas. Assim, considerou-se o indivíduo com saúde boa (autoavaliação muito boa ou boa da saúde na entrevista), regular ou ruim (autoavaliação ruim ou muito ruim da saúde). A variável saúde ruim ficou omitida, como grupo de comparação.

Podem-se resumir os resultados esperados com base na literatura quanto à utilização do PFPB na Tabela 2. Tais sinais se baseiam tanto na utilização ou consumo de medicamentos por meio do sistema público quanto no consumo privado, em que os trabalhos utilizaram diferentes métodos estatísticos e qualitativos para medir a associação entre as variáveis dependentes (que variam de acordo com o trabalho) e o conjunto de características dos indivíduos que utilizam esses medicamentos/programas. Primeiramente, verifica-se que a idade está positivamente relacionada com a utilização de medicamentos, o que pode ter relação com o aumento no número de morbidades com o avanço da idade (Bertoldi et al., 2004). O mesmo resultado foi encontrado nos trabalhos de Arrais et al. (2005) (consumo de medicamentos), Costa et al. (2011) (uso de ao menos um medicamento) e Galvao et al. (2014) (consumo de medicamentos).

Com relação à cor da pele, os resultados encontrados são incertos. Bertoldi et al. (2004) encontraram prevalência de utilização de medicamentos maior entre os homens com pele branca, mesmo após controle para escolaridade e nível econômico. Por outro lado, não foram observadas diferenças para cor da pele entre as mulheres. Por sua vez, Costa et al. (2014) não encontraram significância estatística para cor da pele na prevalência do uso de medicamentos do PFPB entre os que referiram conhecer o programa no município de Campinas (SP) em 2008. Dessa forma, tal variável pode não exercer influência sobre a probabilidade de participação nesse programa.

Verifica-se sinal esperado positivo para a variável que representa as mulheres, o que indica que essa população tem maior probabilidade de demandar medicamentos do PFPB. Esse resultado também foi encontrado nos estudos de Arrais et al. (2005), Costa et al. (2011) e Galvao et al. (2014).

4 Renda baixa foi definida pelo valor de renda per capita igual ou abaixo do percentil 25 (R\$360,00); renda média, pelo valor acima do percentil 25 e igual ou abaixo de 75 (superior a $R \$ 360,00$ e menor ou igual a R\$ 1.200,00); e renda alta, pelo valor de renda per capita acima do percentil de 75 , acima de $\mathrm{R} \$ 1.200,00$.

5 Região Nordeste foi omitida.
Isso aponta para o fato de que é possível que as mulheres se preocupem mais com a saúde do que os homens, fazendo com que elas busquem mais serviços de saúde, como o uso de medicamentos (Chiavegatto Filho et al., 2015). Quando se analisa a relação entre escolaridade e o consumo de medicamentos, não se encontra efeito claro. No estudo de Bertoldi et al. (2004), por exemplo, o efeito da escolaridade desaparece quando se utiliza a regressão ajustada. Galvao et al. (2014) também verificam que, quando os resultados são ajustados pelas covariadas, a educação não se torna associada ao consumo de medicamentos.

Com relação à renda, destacam-se os resultados do estudo de Silva et al. (2012) e Costa et al. (2014), ainda que os primeiros autores não avaliem especificamente classes de renda. No primeiro estudo, os autores analisaram a relação de tal variável com uso diário de dois ou mais medicamentos contínuos em idosos na área urbana no município de Quixadá (CE) em 2009. Os resultados sugeriram associação positiva entre a polifarmácia e renda de mais de um salário mínimo. Porém, no segundo estudo, que tem por foco o uso de medicamentos pelo PFPB, as rendas não demonstraram diferenças estatisticamente significativas. Assim, uma possibilidade é que a renda impacte positivamente a utilização de medicamentos, mas tenha um impacto diferente (ou não impacte) na utilização do programa.

Analisam-se agora as variáveis de saúde e sua relação com a utilização e o consumo de medicamentos. Lima-Costa et al. (2002) verificaram se a cobertura de um plano privado aumenta a utilização de serviços de saúde e o consumo de medicamentos entre idosos no município de Bambuí (MG). Evidenciaram menor utilização de medicamentos prescritos nos idosos que dependiam dos serviços públicos. Já o estudo de Viana et al. (2015) verificou o acesso à medicação continuada entre os idosos no Brasil, por meio dos dados da Pesquisa Nacional por Amostras de Domicílio (PNAD) de 2008. Observam que a possibilidade de comprar medicamento em comparação aos que receberam tudo de graça foi maior nos adultos mais velhos que tinham plano de saúde. Ou seja, apesar de os idosos com plano de saúde consumirem mais medicamentos prescritos, possivelmente é mais fácil que indivíduos sem plano de saúde precisem recorrer a medicamentos gratuitos. Ainda que o PFPB não ofereça apenas medicamentos gratuitos, o sinal do plano de saúde se torna incerto, uma vez que tê-lo pode aumentar o consumo, porém não especificamente de indivíduos que iriam obtê-lo no PFPB.

Em relação à doença crônica, os resultados apresentam elevada robustez. Dessa forma, Costa et al. (2011) e Silva et al. (2012) verificaram relação positiva entre o número de doença crônica e o uso de medicamentos. Já Galvao et al. (2014) analisaram doenças crônicas separadas, encontrando também associação positiva de hipertensão, diabetes, depressão e outras doenças crônicas com o consumo de medicamentos. 
Tabela 2. Sinal esperado das variáveis explicativas utilizadas na presente pesquisa - PNS 2013

\begin{tabular}{lccr}
\hline Características & Variáveis & Resultados esperados & Referências \\
\hline Indivíduo & Idade & Positivo & Costa et al. (2011), Arrais et al. (2005), Galvao et al. (2014) \\
\cline { 2 - 4 } & Branco & Incerto & Bertoldi et al. (2004), Costa et al. (2014) \\
\cline { 2 - 4 } & Pardo & Incerto & Bertoldi et al. (2004), Costa et al. (2014) \\
\hline Educação & Classes de escolaridade & Incerto & Costa et al. (2011), Arrais et al. (2005), Galvao et al. (2014) \\
\hline Renda & Classe média & Incerto & Bertoldi et al. (2004), Galvao et al. (2014) \\
\cline { 2 - 4 } & Classe alta & Incerto & Bertoldi et al. (2004), Silva et al. (2012), Costa et al. (2014) \\
\cline { 2 - 4 } & Plano de saúde & Incerto & Lima-Costa et al. (2012), Costa et al. (2014) \\
\cline { 2 - 4 } & Doença crônica & Positivo & Costa et al. (2011), Silva et al. (2012) \\
\cline { 2 - 4 } & Saúde regular & Negativo & Bertoldi et al. (2004), Silva et al. (2012) \\
\cline { 2 - 4 } & Saúde boa & Negativo & Bertoldi et al. (2004), Silva et al. (2012)
\end{tabular}

Nota: Controles de características geográficas dos domicílios também estão incluídos nas estimações, como número de moradores, região urbana ou rural e regiões brasileiras.

Fonte: Elaboração própria.

Por fim, para classes de autoavaliação de saúde, Bertoldi et al. (2004) verificaram maior prevalência quanto pior a autopercepção. Por sua vez, Silva et al. (2012) agruparam o estado de saúde regular e ruim em uma única variável e encontraram que tal variável estava relacionada positivamente à polifarmácia. Discorre-se ainda que as variáveis de domicílio que incluem número de moradores, urbano e a região do domicílio foram incluídas como controles.

Ainda que não sejam todos os estudos que apresentem por escopo o PFPB, os resultados apresentados são deveras importantes como base para o presente estudo; uma vez expostos, a próxima seção apresenta os resultados encontrados por meio da base de dados da PNS (2013), assim como realiza discussão de tais achados.

\section{Resultados e discussão}

\section{Análise descritiva}

$\mathrm{Na}$ Tabela 3 estão apresentadas as estatísticas descritivas da amostra principal desse trabalho. Conforme discorrido anteriormente, a amostra final foi composta por 9.257 indivíduos, em que 6.831 (74\%) não utilizam os medicamentos do programa. As únicas variáveis não dicotômicas são idade e número de moradores. Em relação à idade, a amostra varia entre os grupos analisados. Nota-se que os não participantes do programa são, em média, mais novos (média $=49,26$ ) do que aqueles que fazem uso dos medicamentos (média $=53,42$ ).

Verifica-se que, com relação às características dos indivíduos, além de o grupo que participa do programa ser formado por pessoas mais velhas, há também diferença estatística na proporção de pardos e negros. No grupo participante, há menos autodeclarados pardos, porém proporção maior de negros. Nas variáveis que captam nível educacional dos amostrados, é possível observar claro padrão nos participantes: geralmente são pessoas com menores níveis educacionais (sem instrução ou ensino fundamental incompleto).

Não foi possível encontrar diferença estatística entre as médias dos grupos não participantes e participantes quando se analisam os indivíduos de classe baixa (renda per capita até $\mathrm{R} \$ 360,00$ ). As diferenças foram observadas, no entanto, nas classes média e alta, em que na primeira há proporção maior no grupo de participantes (média $=57,50 \%$ ) e na classe alta, proporção maior no grupo de não participantes (média = 29,95\%).

Com relação às variáveis relacionadas à saúde da população pesquisada, nota-se que a proporção daqueles que indicaram ter plano de saúde, em geral, é estatisticamente menor no grupo de participantes ( $m e ́ d i a=24,81 \%$ ), como era esperado, uma vez que esse grupo pode ter alguma facilidade de acesso a medicamentes por meio de seu plano. No entanto, quando o respondente da pesquisa indica ter doença crônica ou saúde regular ou ruim, maior é a proporção no grupo de participantes do programa.

Observa-se, ainda, que menor é a proporção de participantes nas regiões Norte e Nordeste brasileira. Apesar de serem regiões com maior vulnerabilidade socioeconômica, a dificuldade ao acesso a farmácias que participam do programa pode ajudar a explicar tal resultado. Na Figura 2, é disposta a proporção de participantes do programa padronizado pela população dessas áreas, assim como a proporção de participantes do PFPB por Unidade da Federação, a partir da amostra final.

Quando se analisou o número de participantes do programa em relação à população de cada estado (sem modificações na base e considerando apenas quem respondeu se pegou ou não medicamentos), os estados que mais se destacaram estão localizados nas regiões Sul e Sudeste. Rio Grande do Sul conta com a maior proporção (3,17\%), seguido por Paraná (2,93\%), Santa Catarina (2,78\%), São Paulo (2,65\%) e Minas Gerais (2,58\%). 
Tabela 3. Estatísticas descritivas das variáveis utilizadas para estimação do modelo Logit - PNS 2013

\begin{tabular}{|c|c|c|c|c|c|}
\hline Características & $\begin{array}{c}\text { Não participantes } \\
(74 \%, n=6.831)\end{array}$ & Desvio-padrão & $\begin{array}{c}\text { Participantes } \\
(26 \%, n=2.426)\end{array}$ & Desvio-padrão & Sig. \\
\hline Idade & 49,26 & 15,82 & 53,42 & 15,23 & $0,00^{*}$ \\
\hline Branco & 43,76 & 49,61 & 45,34 & 49,79 & 0,18 \\
\hline Pardo & 47,06 & 49,92 & 43,94 & 49,64 & $0,01^{* *}$ \\
\hline Negro & 9,18 & 28,87 & 10,72 & 30,94 & $0,03^{* *}$ \\
\hline Mulher & 66,15 & 47,32 & 65,09 & 47,68 & 0,34 \\
\hline Sem instrução & 17,10 & 37,65 & 23,25 & 42,25 & $0,00^{*}$ \\
\hline Fundamental incompleto & 26,73 & 44,26 & 32,89 & 46,99 & $0,00^{*}$ \\
\hline Fundamental completo & 9,05 & 28,69 & 10,18 & 30,25 & 0,10 \\
\hline Médio incompleto & 3,75 & 18,99 & 3,22 & 17,64 & 0,23 \\
\hline Médio completo & 23,07 & 42,13 & 19,95 & 39,97 & $0,00^{*}$ \\
\hline Superior incompleto & 3,75 & 18,99 & 2,23 & 14,76 & $0,00^{*}$ \\
\hline Superior completo & 16,56 & 37,17 & 8,29 & 27,57 & $0,00^{*}$ \\
\hline Classe baixa & 20,74 & 40,55 & 21,06 & 40,78 & 0,74 \\
\hline Classe média & 49,30 & 50,00 & 57,50 & 49,44 & $0,00^{*}$ \\
\hline Classe alta & 29,95 & 45,81 & 21,43 & 41,05 & $0,00^{*}$ \\
\hline Plano de saúde & 35,92 & 47,98 & 24,81 & 43,20 & $0,00^{*}$ \\
\hline Doença crônica ou de longa duração & 39,31 & 48,85 & 48,60 & 49,99 & $0,00^{*}$ \\
\hline Saúde ruim & 15,63 & 36,32 & 19,58 & 39,69 & $0,00^{*}$ \\
\hline Saúde regular & 40,92 & 49,17 & 45,88 & 49,84 & $0,00^{*}$ \\
\hline Saúde boa & 43,45 & 49,57 & 34,54 & 47,56 & $0,00^{*}$ \\
\hline No de moradores & 3,23 & 1,57 & 3,20 & 1,59 & 0,37 \\
\hline Urbano & 83,71 & 36,93 & 82,77 & 37,77 & 0,29 \\
\hline Sul & 15,53 & 36,22 & 19,95 & 39,97 & $0,00^{*}$ \\
\hline Sudeste & 24,64 & 43,09 & 31,20 & 46,34 & $0,00^{*}$ \\
\hline Centro-Oeste & 11,99 & 32,49 & 10,55 & 30,73 & 0,06 \\
\hline Norte & 19,51 & 39,63 & 16,65 & 37,26 & $0,00^{*}$ \\
\hline Nordeste & 28,33 & 45,06 & 21,64 & 41,19 & $0,00^{*}$ \\
\hline
\end{tabular}

Nota: Significância obtida por meio do teste de $t$ em que ** indica que as médias entre os dois grupos analisados são estatisticamente diferentes a $5 \%$ e * a $\%$. Fonte: Resultados da Pesquisa. Elaboração própria.

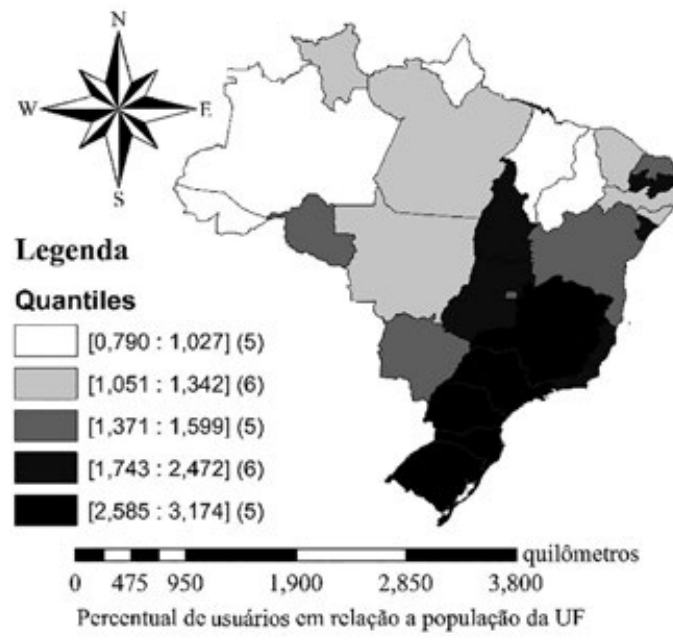

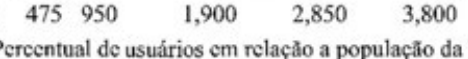

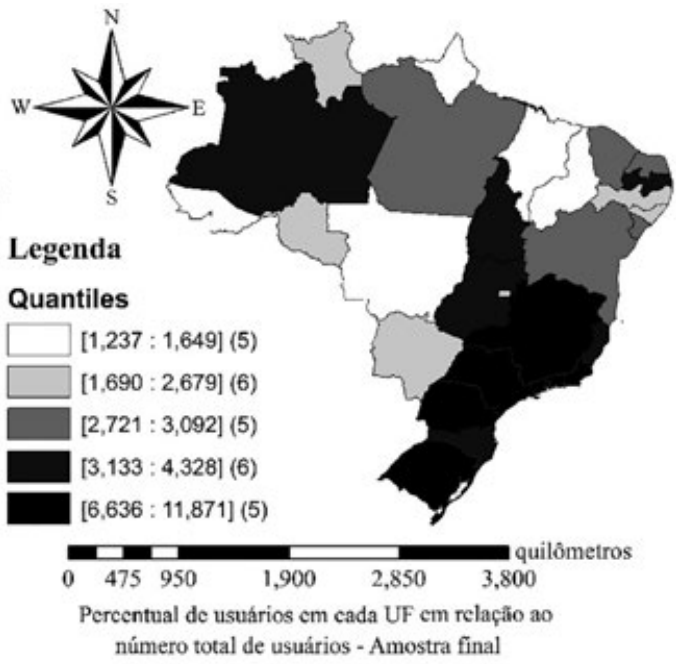

Fonte: Resultados da pesquisa. Elaboração própria.

Figura 2. Quantis da proporção de participantes em Unidades da Federação (UF) em relação ao número do Brasil e da quantidade de participantes padronizados pela população da UF - PNS 2013 
Por sua vez, por meio do segundo mapa da Figura 2, São Paulo é o estado com maior proporção de participantes no PFPB em relação ao total de participantes do Brasil (amostra final). De fato, $11,87 \%$ dos participantes do programa (considerando a amostra final) são residentes desse estado. Percebe-se, no geral, que os estados que agregam o maior número de participantes do PFPB estão mais uma vez na região Sudeste ou na região Sul do Brasil. Apenas os estados de São Paulo, Minas Gerais, Paraná e Rio Grande do Sul correspondem a mais de $36 \%$ dos participantes do programa no Brasil (amostra final).

\section{Resultados econométricos}

Na primeira e na segunda coluna da Tabela 4 estão apresentados os resultados para a estimativa da primeira especificação (conforme seção 3). Verifica-se, para características individuais, que o fato de o indivíduo ser branco e do sexo feminino não impacta significativamente a probabilidade de utilização do PFPB. De fato, ser branco também não demonstrou efeito na prevalência do uso do PFPB no município de Campinas, conforme trabalho de Costa et al. (2014). Apesar de se esperar que mulheres tenham maiores chances de obter medicamentos do PFPB, os resultados obtidos não demonstraram diferença estatisticamente significativa entre homens e mulheres. Resultado similar pode ser encontrado no trabalho de Costa et al. (2016), no qual, apesar de as mulheres obterem mais medicamentos para diabetes e hipertensão no PFPB, não foram encontradas ocorrências de diferenças estatísticas expressivas.

Tabela 4. Estimação do modelo Logit e efeitos marginais para as duas estimações - PNS 2013

\begin{tabular}{|c|c|c|c|c|}
\hline Variáveis & $\begin{array}{c}\text { Utilização } \\
\text { Medicamento PFP }\end{array}$ & $\begin{array}{l}\text { Efeitos } \\
\text { Marginais }\end{array}$ & $\begin{array}{c}\text { Utilização } \\
\text { Medicamento PFP } \\
\text { e Status de saúde }\end{array}$ & $\begin{array}{l}\text { Efeitos } \\
\text { Marginais }\end{array}$ \\
\hline Idade & $0,012^{* * *}$ & $0,002^{* * *}$ & $0,012^{* * *}$ & $0,002^{* * *}$ \\
\hline Branco & $-0,116$ & $-0,021$ & $-0,113$ & $-0,021$ \\
\hline Pardo & $-0,134$ & $-0,025$ & $-0,132$ & $-0,024$ \\
\hline Feminino & $-0,016$ & $-0,003$ & $-0,018$ & $-0,003$ \\
\hline Fundamental incompleto & $-0,077$ & $-0,014$ & $-0,080$ & $-0,015$ \\
\hline Fundamental completo & $-0,056$ & $-0,01$ & $-0,057$ & $-0,011$ \\
\hline Médio incompleto & $-0,185$ & $-0,034$ & $-0,184$ & $-0,034$ \\
\hline Médio completo & $-0,155^{*}$ & $-0,029^{*}$ & $-0,153^{*}$ & $-0,028^{*}$ \\
\hline Superior incompleto & $-0,361^{* *}$ & $-0,067^{* *}$ & $-0,356^{* *}$ & $-0,066^{* *}$ \\
\hline Superior completo & $-0,540^{* * *}$ & $-0,100^{* * *}$ & $-0,531^{* * *}$ & $-0,098^{* * *}$ \\
\hline Classe média & $-0,020$ & $-0,004$ & $-0,020$ & $-0,004$ \\
\hline Classe alta & $-0,265^{* * *}$ & $-0,049^{* * *}$ & $-0,260^{* * *}$ & $-0,048^{* * *}$ \\
\hline Plano de saúde & $-0,402^{* * *}$ & $-0,074^{* * *}$ & $-0,398^{* * *}$ & $-0,073^{* * *}$ \\
\hline Doença crônica/longa duração & $0,249^{* * *}$ & $0,046^{* * *}$ & $0,238^{* * *}$ & $0,044^{* * *}$ \\
\hline Saúde regular & - & - & 0,046 & 0,008 \\
\hline Saúde boa & - & - & $-0,024$ & $-0,004$ \\
\hline No de moradores & 0,005 & 0,001 & 0,006 & 0,001 \\
\hline Urbano & 0,071 & 0,013 & 0,072 & 0,013 \\
\hline Sul & $0,636^{* * *}$ & $0,117^{* * *}$ & $0,639 * * *$ & $0,118^{* * *}$ \\
\hline Sudeste & $0,592^{* * *}$ & $0,109^{* * *}$ & $0,597^{* * *}$ & $0,110^{* * *}$ \\
\hline Centro-Oeste & $0,244^{* * *}$ & $0,045^{* * *}$ & $0,246^{* * *}$ & $0,045^{* * *}$ \\
\hline Norte & $0,151^{*}$ & $0,028^{*}$ & $0,149^{*}$ & $0,027^{*}$ \\
\hline Constante & $-1,715^{* * *}$ & - & $-1,718^{* * *}$ & - \\
\hline Observações & 9.257 & & 9.257 & \\
\hline Prob $>\mathrm{Chi}^{2}$ & 0,0000 & & 0,0000 & \\
\hline Pseudo $R^{2}$ & 0,0408 & & 0,0409 & \\
\hline LROC & 0,6383 & & 0,6387 & \\
\hline Classificação Correta & $73,59 \%$ & & $73,66 \%$ & \\
\hline
\end{tabular}

Fonte: Resultados da Pesquisa. Elaboração própria. Nota: ${ }^{*} p<0,1,{ }^{* *} p<0,05,{ }^{* * *} p<0,01$. 
Com relação à variável idade, observou-se efeito positivo, ou seja, há maior probabilidade de utilizar o PFPB conforme o avanço da idade. À medida que uma pessoa envelhece, seu estoque ótimo de saúde tende a se deteriorar e, em virtude desse fato, a demanda por bens e serviços de saúde (medicamentos, exames clínicos, consultas médicas, entre outros) tende a aumentar ao longo da vida (Grossman 1972; 1976; 2000). Tal resultado também está de acordo com os encontrados em Costa et al. (2011), Arrais et al. (2005) e Galvao et al. (2014).

Para analisar como os níveis diferentes de escolaridade afetam a probabilidade de participação no programa, utilizaram-se como grupo de referência indivíduos sem nenhuma escolaridade. O sinal esperado por tais variáveis é incerto, já que após algum tipo de ajuste tais variáveis perderam seu efeito (Bertoldi et al., 2004; Galvao et al., 2014).

Ainda assim, de acordo com os resultados, percebe-se que apenas os grupos com ensino médio completo ou algum grau de ensino superior se diferenciam estatisticamente do grupo sem escolaridade, em que ter maior nível educacional reduz a probabilidade de uso do programa. Esse resultado pode ser explicado pela importância que a educação possui para a procura por informações associadas às doenças e suas prevenções, busca por hábitos mais saudáveis e pela utilização mais eficiente dos serviços e cuidados com a saúde (Göpffarth et al., 2016).

Para as variáveis de renda, verificou-se que estar inserido na classe de renda alta (em comparação com a classe de renda baixa) reduz a probabilidade de obtenção de medicamentos pelo PFPB. Esse resultado está de acordo com a literatura acerca da composição do gasto familiar brasileiro. De acordo com Garcia et al. (2013), com dados da Pesquisa de Orçamento Familiar (2002-2003 e 2008-2009), predominam entre as famílias de maior renda gastos com medicamentos para hipertensão, diabetes e doenças do coração. Por sua vez, as famílias de menor renda tendem a direcionar seus gastos em medicamentos analgésicos antigripais e anti-inflamatórios. Ou seja, famílias de menor renda necessitam mais dos medicamentos-alvo do programa, já que famílias de maior renda apresentam maior acesso a eles. $O$ resultado, porém, vai na contramão ao apresentado por Costa et al. (2014), em que não se verificou diferença significativa na prevalência do uso de medicamentos pelo PFPB, porém tal estudo baseia-se em uma amostra com enfoque no município de Campinas (SP).

Por sua vez, a variável "plano de saúde" também apresentou diminuição na probabilidade de obtenção de medicamentos do PFPB. Tal resultado pode estar relacionado com o trabalho de Viana et al. (2015), o qual indica que adultos mais velhos que possuem planos de saúde apresentam maior possibilidade de comprar medicamentos, em comparação aos indivíduos que recebem de graça, ainda que, de fato, nem todos os medicamentos do PFPB sejam obtidos de forma gratuita.

A variável doença crônica mostrou efeito marginal positivo e significativo em obter medicamentos pelo PFPB. Essas enfermidades têm provocado elevado número de mortes prematuras e queda da qualidade de vida e afetado de maneira negativa as esferas sociais e econômicas de países desenvolvidos e em desenvolvimento (WHO, 2014). Tais resultados estão de acordo com os estudos de Costa et al. (2011), Silva et al. (2012) e Galvao et al. (2014). De acordo com Almeida et al. (2019), a ampliação de medicamentos para hipertensão arterial e pelo programa foi fundamental para a redução de internações e óbitos decorrentes desses agravos.

Na terceira e na quarta coluna da Tabela 4, estão apresentados os resultados para a estimativa da segunda especificação (conforme a seção 3). De maneira geral, acrescentou-se à análise a percepção de saúde do indivíduo. Verificou-se que o status de saúde não foi significativo, demonstrando que a percepção da saúde do indivíduo ser boa ou ruim não impacta sobre a utilização dos medicamentos do programa. Esperava-se que tais variáveis apresentassem sinal negativo e estatisticamente significativo, conforme resultados de Bertoldi et al. (2004) e Silva et al. (2012). Porém, uma questão mais importante pode estar relacionada com o tipo de diagnóstico do indivíduo, por exemplo, ter sido diagnosticado com doença crônica ou de longa duração, que novamente impactou positiva e significativamente sobre a utilização dos serviços.

Além disso, ter maiores níveis de escolaridade e estar inserido em níveis mais alto de renda continua a ser significativo e a apresentar efeitos marginais negativos, demonstrando que, no geral, indivíduos que utilizam os medicamentos do programa acabam por ser aqueles que, de fato, necessitam deles, principalmente com relação à renda. Destaca-se, porém, que alguns participantes que teoricamente necessitariam menos dos medicamentos, sejam eles gratuitos ou com desconto, como os que apresentam maior renda, também os utilizam.

\section{Considerações finais}

Este estudo analisou os fatores associados à probabilidade de utilização do PFPB, a partir dos dados da PNS de 2013. Aplicou-se o modelo econométrico Logit em duas diferentes especificações. A primeira analisou características individuais, de renda, educacionais, domiciliares e o diagnóstico de doença crônica. Na segunda especificação, além dos fatores mencionados, consideraram-se as respostas subjetivas de status de saúde. Por fim, vale destacar que em ambas as estimações se utilizaram as regiões brasileiras como variáveis de controle.

Identificou-se que apresentar diagnóstico de doença crônica (ou de longa duração) e avanço da idade e não ter 
plano de saúde aumenta a probabilidade de utilizar os benefícios do PFPB. Observou-se, também, que ter faixas maiores de escolaridade e pertencer a classe de renda alta impacta negativamente o uso do programa. Por sua vez, as variáveis referentes a raça e sexo feminino não apresentaram sinal estatisticamente significativo. Com relação à segunda especificação, não foi verificado impacto significativo das variáveis de status de saúde sobre o consumo do programa.

A utilização do PFPB está associada aos fatores socioeconômicos e de morbidade, o que demonstra a importância de políticas de assistência farmacêutica no processo saúde/doença. Ainda que alguns indivíduos usem o programa sem precisar, esse ofereceu uma alternativa importante na obtenção de medicamentos para indivíduos inseridos nas camadas mais baixas de renda e que apresentam diagnóstico de doenças crônicas. Essas informações permitem que os gestores públicos tenham uma maior compreensão do uso do programa pela população, possibilitando melhor direcionamento, ampliação e qualificação do acesso aos medicamentos.

Diante do exposto, este trabalho contribuiu para uma literatura ainda incipiente sobre o entendimento dos determinantes da utilização do PFPB e levanta questões importantes para pesquisas futuras. Recomenda-se, por exemplo, incluir mais variáveis de controle e utilizar outros recortes amostrais para confirmação dos resultados, já que foram considerados na amostra final apenas indivíduos chefes do domicílio ou cônjuges do sexo oposto. Algumas questões importantes não foram avaliadas, como, por exemplo, se os medicamentos foram gratuitos ou com necessidade de copagamento, assim como se o paciente deu continuidade ao tratamento, já que a pergunta se refere apenas ao último atendimento de saúde.

\section{Agradecimentos}

Os autores agradecem à Coordenação de Aperfeiçoamento de Pessoal de Nível Superior (Capes) pelas bolsas de estudos (doutorado) concedidas a Pietro André Telatin Paschoalino, Wander Plassa e José Rodrigo Gobi durante a realização desta pesquisa.

\section{Referências bibliográficas}

Almeida ATCD, Sá EBD, Vieira FS, Benevides RPDS. Impacto do Programa Farmácia Popular do Brasil sobre a saúde de pacientes crônicos. Rev Saúde Pública. 2019;53.

Arrais PSD, Brito LL, Barreto ML, Coelho HLL. Prevalência e fatores determinantes do consumo de medicamentos no Município de Fortaleza, Ceará, Brasil. Cad Saúde Pública. 2005;21:1737-46.

Arvate P, Biderman C. Economia do setor público no Brasil. Brasil: Elsevier; 2013.

Bertoldi AD, Barros AJD, Hallal PC, Lima R. Utilização de medicamentos em adultos: prevalência e determinantes individuais. Rev Saúde Pública. 2004;38:228-38.
Brasil. Decreto n 5.090, de 20 de maio de 2004. Regulamenta a Lei 10.858, de 20 de maio de 2004, institui o programa "Farmácia Popular do Brasil", e dá outras providências. Diário Oficial da União, Poder Executivo, Brasília, DF, n. 97, seção 1, p. 6, 21 maio 2004. Disponível em: http:// www2.camara.leg.br/legin/fed/decret/2004/decreto-5090-20-maio2004-532380-norma-pe.html. Acesso em: 29 jan. 2019.

Brasil. Ministério da Saúde. Farmácia Popular: Sobre o programa. 2020a. Disponível em: http://portalms.saude.gov.br/acoes-e-programas/ farmacia-popular/como-utilizar. Acesso em: 6 jan. 2020.

Brasil. Ministério da Saúde. Farmácia Popular: Sobre o programa. 2020b. Disponível em: http://portalms.saude.gov.br/acoes-e-programas/ farmacia-popular/sobre-o-programa. Acesso em: 6 jan. 2020.

Brasil. Ministério da Saúde. Fundação Oswaldo Cruz. Programa Farmácia Popular do Brasil: Manual Básico. Brasília: Ministério da Saúde; 2005. Disponível em: http://portalarquivos2.saude.gov.br/images/pdf/2014/ abril/23/manual-basico-fp1170511.pdf. Acesso em: 24 abr. 2019.

Brasil. Ministério da Saúde. Lista de medicamentos disponibilizados pelo "Aqui tem farmácia popular". 2018a. Disponível em: http:// portalarquivos2.saude.gov.br/images/pdf/2019/janeiro/07/ListaMedicamentos.pdf. Acesso em: 6 jan. 2020.

Brasil. Ministério da Saúde. Portaria no 491, de 9 de março de 2006. Dispõe sobre a expansão do Programa "Farmácia Popular do Brasil". Diário Oficial da União, Poder Executivo, Brasília, DF, n. 48, seção 1, p. 59, 10 mar. 2006. Disponível em: http://bvsms.saude.gov.br/bvs/saudelegis/ gm/2006/prt0491_09_03_2006_comp.html. Acesso em: 6 jan. 2020.

Brasil. Ministério da Saúde. Secretaria de Ciência, Tecnologia e Insumos Estratégicos. Departamento de Assistência Farmacêutica e Insumos Estratégicos. Assistência Farmacêutica no SUS: 20 anos de políticas e propostas para desenvolvimento e qualificação - relatório com análise e recomendações de gestores, especialistas e representantes da sociedade civil organizada. Brasília: Ministério da Saúde; 2018b.

Cameron AC, Trivedi PK. Microeconometrics: methods and applications. New York: Cambridge University Press; 2005.

Chiavegatto Filho ADP, Wang YP, Malik AM, Takaoka J, Viana MC, Andrade LH. Determinantes do uso de serviços de saúde: análise multinível da Região Metropolitana de São Paulo. Rev Saúde Pública. 2015;49:1-12.

Conasems - Conselho Nacional de Secretarias Municipais de Saúde. Nota Informativa no 18/2017. 2017. Disponível em: https://www.conasems. org.br/wp-content/uploads/2017/04/Nota-encerramento-RedePr\%C3\%B3pria-FPB.pdf. Acesso em: 6 jan. 2020.

Conass - Conselho Nacional de Secretários de Saúde. Programa Farmácia Popular do Brasil. 2016. Disponível em: http://www.conass.org.br/wpcontent/uploads/2017/01/2-b.pdf. Acesso em: 2 jul. 2018.

Costa KS, Barros MB, Francisco PM, César CL, Goldbaum M, Carandina L. Use of medication and associated factors: a population-based study in Campinas, São Paulo State, Brazil. Cad Saúde Pública. 2011;27(4):649-58.

Costa KS, Francisco PMSB, Barros MBA. Conhecimento e utilização do Programa Farmácia Popular do Brasil: estudo de base populacional no município de Campinas-SP. Epidemiol Serv Saúde. 2014;23(3):397-408.

Costa KS, Tavares NUL, Mengue SS, Pereira MA, Malta DC, Silva Júnior JB. Obtenção de medicamentos para hipertensão e diabetes no Programa Farmácia Popular do Brasil: resultados da Pesquisa Nacional de Saúde, 2013. Epidemiol Serv Saúde. 2016;25:33-44.

Galvao TF, Silva MT, Gross R, Pereira MG. Medication use in adults living in Brasilia, Brazil: a cross-sectional, population-based study. Pharmacoepidemiol Drug Saf. 2014 May;23(5):507-14.

Garcia MM, Guerra Júnior AA, Acúrcio FA. Avaliação econômica dos Programas Rede Farmácia de Minas do SUS versus Farmácia Popular do Brasil. Ciênc Saúde Coletiva. 2017;22(1):221-33. 
Garcia LP, Santanna AC, Magalhães LCGD, Freitas LRSD, Aurea AP. Gastos das famílias brasileiras com medicamentos segundo a renda familiar: análise da Pesquisa de Orçamentos Familiares de 2002-2003 e de 2008-2009. Cad Saúde Pública. 2013;29(8):1605-16.

Göpffarth D, Kopetsch T, Schmitz H. Determinants of regional variation in health expenditures in Germany. Health Econ. 2016;25(7):801-15.

Greene WH. Econometric analysis. Delhi: Pearson Education India; 2011.

Grossman M. On the concept of health capital and the demand for health. J Polit Econ. 1972;80(2):223-55.

Grossman M. The correlation between health and schooling. In: Terleckyj NE, editor. Household production and consumption. New York: National Bureau of Economic Research; 1976.

Grossman M. The human capital model. In: Culyer AJ, Newhouse JP, editor. Handbook of health economics. Amsterdam: Elsevier Science; 2000.

IBGE - Instituto Brasileiro de Geografia e Estatística. Pesquisa Nacional de Saúde. Disponível em: http://www.ibge.gov.br/home/estatistica/ populacao/pns/2013/default.shtm. Acesso em: 20 jun. 2018.

Lima-Costa MFF, Guerra HL, Firmo JOA, Vidigal PG, Uchoa E, Barreto SM. The Bambuí Health and Aging Study (BHAS): private health plan and medical care utilization by older adults. Cad Saúde Publica. 2002;18(1):177-86.

Motta GP, Domingues EP, Andrade MV, Chein F, Santiago FS. Uma análise dos impactos econômicos do Programa Farmácia Popular do Brasil. In: 410 Encontro Nacional de Economia - ANPEC, Foz do Iguaçu; 2013. Disponivel em: https://www.anpec.org.br/encontro/2013/files_l/i12-e75 ad295e5f96a014e11b00bd2226ab7.pdf. Acesso em: 17 nov. 2020.
Pereira MA. Programa Farmácia Popular no Brasil: uma análise sobre sua relação com o Complexo Econômico-Industrial da Saúde e os programas estratégicos do Governo Federal [dissertação de mestrado]. Rio de Janeiro: Escola Nacional de Saúde Pública Sergio Arouca (ENSP)/ Fundação Oswaldo Cruz (Fiocruz); 2013.

Plassa W, Cunha MS. Sensação de insegurança pública no Brasil: uma análise estrutural das vulnerabilidades e do efeito da vitimização direta. Economic Analysis of Law Review [revista eletrônica]. 2016;7(1):266-90. Disponível em: https://portalrevistas.ucb.br/index.php/EALR/article/ view/6818. Acesso em: 17 nov. 2020.

Santos-Pinto CDB, Costa NDR, Osorio-de-Castro CGS. Quem acessa o Programa Farmácia Popular do Brasil? Aspectos do fornecimento público de medicamentos. Ciênc Saúde Coletiva. 2011;16(6):2963-73.

Silva GOB, Gondim APS, Monteiro MP, Frota MA, Meneses ALL. Uso de medicamentos contínuos e fatores associados em idosos de Quixadá, Ceará. Rev Bras Epidemiol. 2012;15(2):386-95.

Silva RM, Caetano R. Programa "Farmácia Popular do Brasil": caracterização e evolução entre 2004-2012. Ciênc Saúde Coletiva. 2015;20(10):2943-56.

Viana KP, Brito AS, Rodrigues CS, Luiz RR. Access to continued-use medication among older adults, Brazil. Rev Saúde Pública. 2015;49(14).

WHO - World Health Organization. Global status report on noncommunicable diseases: 2014. Geneva: WHO; 2014. 